\title{
Contextual Information Integration Platform for Humanitarian Relief
}

\author{
Shivsubramani Krishnamoorthy \\ MIND Lab, Department of Computer Science, \\ University of Maryland \\ College Park, MD 20742. USA \\ shiv@cs.amrita.edu
}

\author{
Ashok Agrawala \\ MIND Lab, Department of Computer Science, \\ University of Maryland \\ College Park, MD 20742. USA \\ agrawala@cs.umd.edu
}

\begin{abstract}
It is well recognized that context plays a significant role in all human endeavors. All decisions are based on information which has to be interpreted in context. By making the information systems context-aware we can have systems that significantly improve the human capabilities to handle situations context. In this paper, we highlight the significance of Context that wireless devices should be able to support, especially in the event of a calamity or a disaster to provide much more effective support to the relief efforts. In such situations, where wireless device users need to be provided with critical information for purposes such as evacuation, relief work etc., it is equally important to provide information that suits current situation of the user while taking into account the current environment around the user. We present Rover 2.0, framework for contextual information integration for wireless devices. With our belief that information processing systems should only provide support to the human decision maker (and not attempt to replace him/her), the Rover provides means for supporting the flow of information filtered and augmented using relevant contextual information. Our framework uses a paradigm for handling context information that includes user specific context combined with activity context and common context. We outline the significance of context-awareness with the help of a mobile application/system developed at Maryland on how effective such systems can be in situations of disaster or calamity.
\end{abstract}

\section{Keywords-Context, Context-Aware, Rover2.0, M-Urgency}

\section{INTRODUCTION}

Today, we see a trend in which wireless devices have penetrated into the society and mobile phones are being used by a significant portion of the population not only in developed countries but also in developing countries. It is well recognized that the use of cell phones is having a far reaching positive economic impact on society. This impact is for day to day activities and is a result of the easy availability and accessibility of information. Only raw information is available as the majority of phones has limited processing and storage capabilities and cannot provide the contextual information which is most useful for its users. The basic purpose of such device is simply to transmit or receive information which may appear in text, audio, video, sensor day or some other such form.
The most useful form of information is when its context is available with it. In most situations the information context is available locally. In order to make sense about a larger area or larger collection of information it has to be collected at one point so that suitable integration can be done. Clearly the movement of information requires communications facilities and integration requires suitable storage and processing facilities. This is especially true in a disaster situation in which the communication infrastructure may be damaged and the processing facilities may not be fully functional. The roles that wireless networks play, then, assume a significant importance. Such networks have to adjust and adapt to the available resources/connectivity etc. and provide an infrastructure that can be used for information integration and situational awareness.

Note that the role of information integration is not only in creating a better situational awareness at the point of integration but also the ability to disseminate the information to the people in the field and to the resources available at divergent locations to address the needs. This step also requires communication.

In this paper we focus on the problems of information integration and present Rover 2.0, a context-aware information integration platform for wireless devices. It is an ongoing effort to cater mobile devices in storing, managing and utilizing contextual information based on the situation in which they are used.

We also describe M-Urgency, a public safety system developed by us on the Rover platform that redefines how emergency calls are made to first responders such as the police department. M-Urgency is a good example that establishes the effectiveness of a context aware system/application in situations of humanitarian crisis or a calamity.

\section{A. Motivation}

When an earthquake struck the east coast of the United States one afternoon in August 2011, everybody was totally caught unprepared for a situation. The first reaction of people was to call home to tell their loved ones that they are alright and to inquire about their safety. As a consequence so many people tried to use their cell phones that the phone 
system got congested and it took some people hours to get through. Also, streets got flooded with people in no time; highways got totally congested with everyone trying to drive back home when the offices were closed early. A situation of chaos followed. Further, in a few days Hurricane Irene was to hit the region and preparations began for that. People were still uncertain about what if an earthquake aftershock occurred when the hurricane struck. People had some precautionary instructions for each of the | calamity but were unaware of the steps to be taken when unexpected situations occur.

Clearly an infrastructure is required in which the context of the situation is understood (more effectively during unexpected developments that are common during calamities) along with the context of each user, and incidence, and relevant critical information is made available to him/her. We believe taht any crisis management will be ineffective if relevant, critical and timely information is not provided on demand.

\section{B. Objective}

Addressing such situations we discuss in this paper our initial attempts towards effectively managing contextual information in situation of crisis. In this paper, we:

- Highlight the significance of contextual information available to people in situations of humanitarian crisis or calamity

- Introduce Rover 2.0, a contextual information integration platform for wireless devices

- Present M-Urgency, a public safety emergency application that proves the effectiveness of contextual information in case of emergency and discuss how such a system can be extended to handle more complex situations.

\section{Overview}

We have organized the paper as the following. In section 2 we discuss related work, on how the significance of context for humanitarian relief planning has been discussed in the literature. We provide a general discussion about context and context-awareness in section 3. We describe the Rover 2.0 architecture in section 4. Section 5 presents the M-Urgency architecture and discusses how an extension or a variant of the application can be effectively used in a situation of humanitarian crisis or calamity.

\section{RELATED WORK}

\section{A. Context-awareness for Humanitarial relief planning}

A lot has been discussed in the literature on how context of the situation and place is relevant when humanitarian relief is planned. The paper [1] reflects on how local area details provide the context to which plans are tailored. Rainsford et. al. [2] highlights how information overloading is avoided if information is provided to the user in specific context.
Barani et. al [3] specifically stress upon context-awareness when collaborative planning is done between distributed planners. Kikiras et. al. [4] has proposed an integration platform for autonomic computing for disaster relief. The idea here is to provide a middleware for intelligent, collaborative and context aware services using rumor spreading techniques. Siren [5] provides a foundation for gathering, integrating, and distributing contextual data, such as location and temperature to support tacit communication between firefighters with multiple levels of redundancy in both communication and user alerts. Tomaszewski et. al. [6] says information foraging and sensemaking with heterogeneous information are context-dependent activities. They attempt the challenges of constructing and representing context within visual interfaces that support analytical reasoning in crisis management and humanitarian relief. The paper[7] specifically discuss about geo-spatial information. They briefly put forth on how groups or groups of groups can work with geospatial information and technologies in crisis management.

\section{B. Context-Aware or Integration Frameworks}

Here, we briefly discuss the developments in the field of context aware systems discussed in the literature.

Dey et al [8] defined a context-aware system as a system that uses context to provide relevant information and/or services to the user where relevancy depends on the users task. They focus on adaptiveness of the application rather than change in its behavior. They also specify certain features that a context-aware application should support: presentation of information and services to a user, automatic execution of a service for a user and tagging of context to information to support later retrieval. Bolchini et al [9] identify the following categories for models and contextaware systems based on the main focus of the model, the representation of context and the usage of context:

- Channel-device-presentation - This category of context-aware systems are application centric, with limited management of location and time. They have limited flexibility and are characterized by centrally defined context.

- Location and environment - These models provide accurate location and time management, high degree of flexibility and centralized definition of context.

- User activity - This category of models focuses on the user and the user's activity as the main subject.

- Agreement and sharing of distributed context These focus mainly on information and context sharing. Context definition is distributed and context quality monitoring and ambiguity are the key issues.

- Selecting relevant data, functionalities and services These cater towards using context to determine which information, functionalities and services are relevant to the user. The application is the main 
subject of the model and context dimensions like time and location are accurately provided.

A context-aware system that captures all the features of these aforementioned categories will focus on the context problem as a whole, and will be abstract and generic enough to be applicable in any domain or environment. Some of the contemporary context models and context-aware systems, which satisfy this requirement, include ACTIVITY [10], an Activity Theory based model which encapsulates context as a set of elements which influence users intentions while doing some activities. The model appears to be in its nascent stage and the implementation details are not very lucid. Another such system is described in [11], which has a conceptual framework that considers context as a part of a process rather than a state. In this system, context includes all the entities, their roles, the relations between the entities and the situations of the entities. In [12], Yang et al, describe an ontology based context model which is specific to learning environments.

The context-aware integration framework that we present in this paper is designed not to be specific to any domain but to approach the context problem from all perspectives. It falls into all the categories that have been mentioned in [9].

\section{SIGNIFICANCE OF CONTEXT-AWARENESS IN HUMANITARIAN CRISIS}

Most of the natural calamities occur unexpectedly with very little warning. Before the calamities all the resources that may be needed to address the situation are located at their normal locations for storage. The situation on the ground varies widely in the area and so do the needs for help, whether it is for putting out fire, rescue from collapsed building, flood, trapped in vehicle etc., emergency medical evacuation support, transportation food shelter, etc. All such needs are only known in the local area and the information about the need, in context of the local area and its timelyness have to be available for coordination.

In most calamities the resources needed far exceed the requirements and most of the resources are not at the places where they are needed. In order to effectively organize the support operation the first requirement is that of the ground truth reflecting not only the needs at various locations but also the context including the urgency of support. The knowledge about the resources of various kinds is also needed. Then only any suitable plan can be prepared and executed in a coordinated manner. When such steps are not taken chaos often results.

We note that getting the right information from various locations to the location where coordination can be carried out requires the movement of a lot of information. As the infrastructure for communication is often affected by such calamities, wireless communications which can continue to function play a very significant role.

Further, most of the help is provided to people affected by the calamity and the individuals not only need help but also information about the situation around them as well as about their loved -ones.

Thus, we believe that critical information provided by/to the people in case of emergencies or crises should not just be timely, but should be relevant to the person. This can be done only if the context of the person is understood along with the context of the situation/environment around him. Timely and critical decisions are possible based on how effective the piece of provided information is.

\section{A. Information Integration Point}

When an event happens, we require an "Information Integration Point" (IIP) where the information acquired from varied sources can be compiled meaningfully together and effectively provided to the different categories of people - be it the general public which is getting effected or the personnel who are addressing the situation of calamity from the administration side.

We propose an Information Model as shown in figure 1, which depicts a continuous series of operations, or process, whose performance is enhanced at each node, and collectively through mutual access to information and collaborative utilization of information.

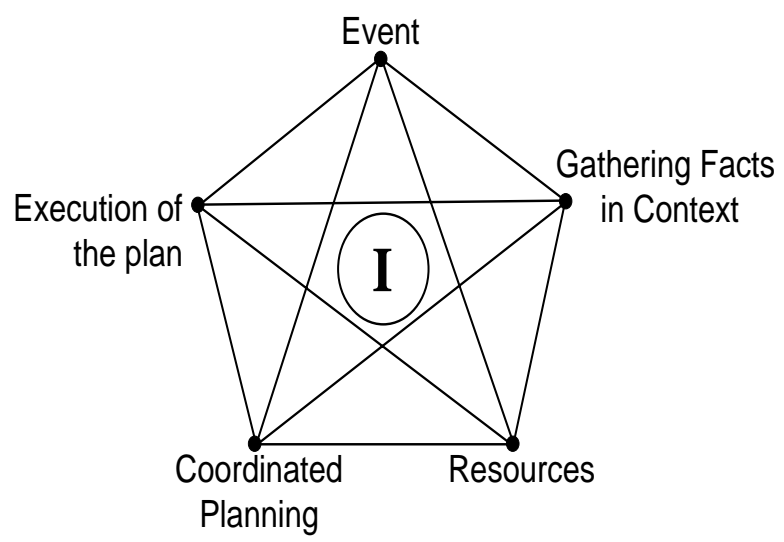

Fig 1: Information Model for effective information management during an event.

The "I" in the information model represents the information pool that is generated as the consequence of an event. By event we mean a natural calamity or a situation of humanitarian crisis. A series of activities or processes follow the event, which are represented by the rest of the nodes. The nodes revolve around information, either utilizing it or contributing to it. The nodes are inter-related with each other 
through the information they share, one process complementing the other.

As the event takes place, all the information regarding the event can be collected and pooled at the IIP. It is critical that that information is gathered in context, based on the situation and relevance. For example, when an earthquake tremor is felt in a particular city, information such as the weather, traffic in major roads, information related to the population of the city may all be relevant. But information such as the literacy rate or birth rate with respect to the city does now have much relevance in addressing the situation at hand.. Thus, the facts are to be gathered in context at the IIP. The gathered information could be of two types:

- Static Contextual Information: Information that would remain unchanged over the period the relief work is on. For example, the information related to the offices and how many people work at each, the location and normal capabilities of the hospitals, etc..

- Dynamic Contextual Information: Information that change rapidly as the various activities are initiated. For example, the road traffic situation, weather etc.

The next step would be to gather the information about all the various resources available that need to be utilized as part of the relief work. A coordinated planning follows based on the information available about the event, the contextual related information regarding the event and the information about the resources. There may be many bodies/organizations involved in the relief work. Thus, a coordinated effort has to be facilitated by the IIP. IIP would be the core of the activities where the key decisions are made by personnel based on the different forms of information available. Then, the IIP is responsible in initiating the execution of the plan.

This model is incomplete with just a single iteration of the above mentioned steps. As discussed before, an event will lead to more consequential events for which the whole cycle has to be repeated again. As discussed in the beginning of this section, though the earthquake was the major event, it incited more events like situations of major traffic jam, water pipeline damage which require a full iteration in the information model each.

We also note that in a relief operation there may be multiple IIPs such that each may have some dedicated responsibilities and there may be one IIP which coordinates the efforts of other IIPs.

\section{B. Means of Communication}

We see the trend today where smartphones are on a surge. It is believed that smartphones will outnumber the feature phones by 2011 in the US [13]. Along with the provisions for communication, they also carry many sensors these days like GPS, high resolution cameras, accelerometer etc. These sensors could prove useful in recording contextual information. With these wireless devices having penetrated the masses, it could prove to be the most effective means of communicating critical and contextual information to the masses. They could also be used as means of gathering the context of the situations effectively as they are spread over different places.

\section{ROVER 2.0 ARCHITECTURE}

To take our arguments, in the previous section, to a practical level, we propose Rover 2.0, which would act as the information integration point (IIP), represented by "I" in the information model.

Rover 2.0 is an integration and fusion platform that seeks to provide a mechanism to aid communication of heterogeneous information sources to all interested and authorized entities. Additionally, entities utilizing Rover add as much information as possible to aid in the fusion of information sources, including augmenting information sources with additional contextual information. Rover can provide transformations for all sorts of information, from the context and content from different types of sources, into an appropriate view for relief operation.

Rover 2.0 caters to the development of context-aware applications. It not only provides means to store and retrieve context information, but also facilitates relevant services to the applications so that the context information could be more effectively used. Rover 2.0 implementation is an advance on Rover [14][15], where some aspects of the existing architecture were revisited. Figure 2 provides a high level layout of the Rover 2.0 architecture. This architecture is centered on the information flow between the various sources and the end application or the user. The architecture is organized in three tiers as presented below:

\section{Service Tier}

The service tier is implemented as a collection of service engines and Figure 1 shows some of the service engines that have been implemented in the system to date. The key role of the service engines is to fetch or to translate unstructured information, to meaningful and useful form for the user, and provide services as needed. We have implemented three services at this time.

The location service plays the role of resolving a GPS or Wi-Fi based coordinates to actual physical location; The streaming service enables any application to establish an audio/video stream with the system; The external service enables incorporation of external information to add to the clarity of the context, e.g. the weather information, traffic information etc. We are in the process of adding more services to this tier.

\section{Interface Tier}

This tier provides a well defined interface for the clients/applications to associate with the system, though API calls. 


\section{Context Tier}

This is the critical part of the system which makes use of the context information of each user to mediate the flow of information from the source to the client/user. Based on the contexts of the user, along with the general context of the environment, the information is filtered, adjoined and/or rearranged, and some services enabled.

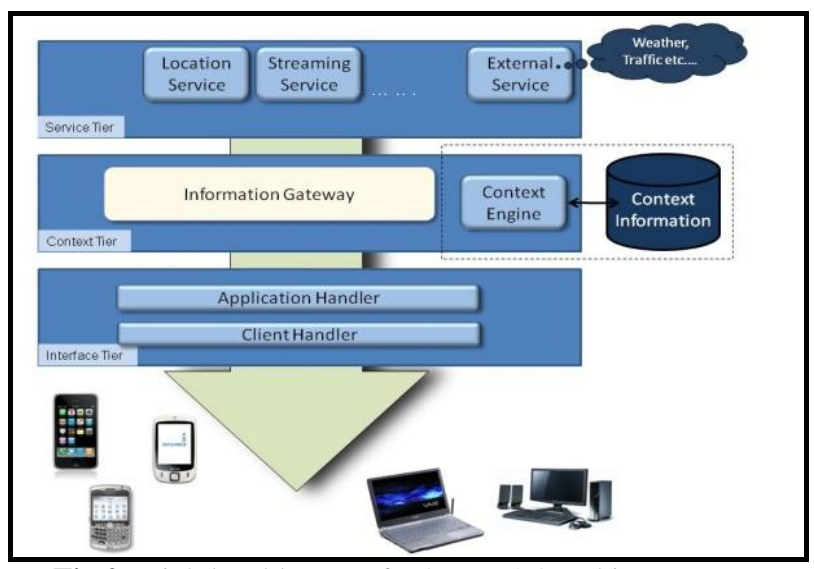

Fig 2: High level layout of ROVER 2.0 architecture

Rover 2.0 is still under development. Here we have discussed the components that are fully or partially functional. It has been designed and being developed in such a way that it is extensible and provides the application developers means to add service engines to the service tier to cater to their specific needs. The design supports distributed operation.

Any wireless device with the capability to transmit data over the internet will be capable of availing the provisions in Rover. Identifying mobile phones as more prevalent wireless devices, we have developed a suite of mobile applications, caller $M y e V y u$, on Rover that are rooted on efficiently managed contextual information. M-Urgency is a public safety system from this suite which we present next.

\section{M-URGENCY}

In this section we provide a public safety system developed by us that handles situations of emergencies that an individual or a group of people may encounter. We believe that this example application is a strong proof of how effective and efficient emergency handling can become if the right contextual information is available at the point of decision making and if convenient methods are provided to them to initiate the activities. Though this system is not designed to address large scale emergencies in the event of a natural calamity or humanitarian crisis, we strongly believe that $\mathrm{a}$ it proves to be a promising basis which can lead to a system that can handle a major situation of emergency, caused by a disaster or calamity.

M-Urgency $^{1}$ [16][17] is a public safety application which is a significant advance in how emergency calls are handled. M-Urgency enables a person to establish and audio and a video stream connection with a Public Safety Answering Point (PSAP), to provide the most precise idea about the situation, along with contextual information including his real-time location, disabilities, special needs etc. Since the emergency personnel are provided with all the contextual information, they are in a position to efficiently provide service accordingly. Further, M-Urgency enables the dispatcher to forward the stream and the contextual information to first responders like a police officer, ambulance, fire engine etc., assigned to handle the call, by a gesture as simple as drag and drop. In this way the responder knows the latest situation as he/she travels to the incident scene.

\section{A. Components}

There are various components of M-Urgency system that communicate with Rover 2.0.

\section{Caller Application}

It runs on a Smartphone, tablets, PDA, laptop, etc., allowing the user to initiate an emergency call by the press of a button. The application communicates with Rover 2.0 and facilitates the location and the streaming service. Thus, an audio/video stream along with the location information is sent to the PSAP. Fig 3 shows the screenshot of the caller application.

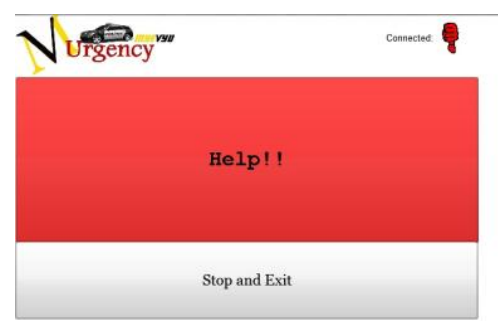

Fig 3: Caller Application screenshot

\section{Dispatcher Console Application}

This is the critical part of the M-Urgency system. Used by the PSAP, it runs on a desktop and receives emergency calls with the audio/video feeds. Fig 4 shows the screenshot of the dispatcher console application. It enables the dispatcher to:

- View the incoming calls (on the left) and interact with them using audio selectively as needed,

- View the real time location of the caller on a map interface along with the context information about the caller,

\footnotetext{
${ }^{1}$ http://m-urgency.umd.edu
} 
- View the list of available emergency responders (on the right),

- Group more than one emergency calls together, if so desired,

- Assign responder(s) to the caller/incident through drag and drop operations, etc.

We present the actual sequence of events in the subsequent sections.

\section{Emergency Responder Application}

It runs on laptops, typically in the emergency vehicle. Once the dispatcher assigns the responder to an incident/caller, this application receives the forwarded audio/video streams of the assigned caller(s) along with the real time location information of the same. Thus the responder gets the precise information about the emergency situation and can get there prepared accordingly. Fig 5 shows the screenshot of a responder application.

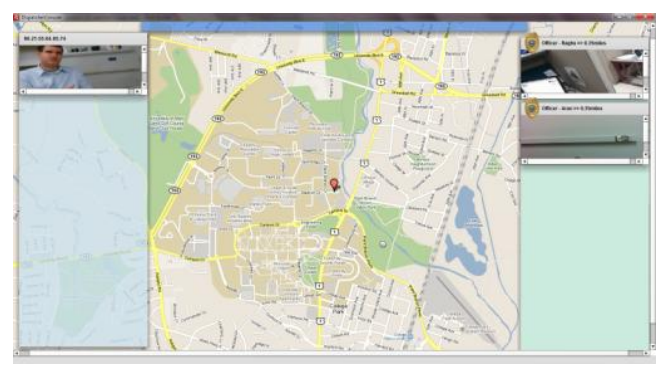

Fig 4: Dispatcher console application screenshot

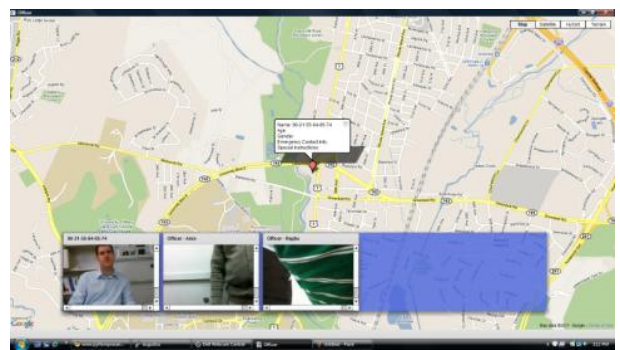

Fig 5: Emergency Responder application screenshot

In practical situations, the emergency responder cannot always be present in the emergency vehicle to receive the calls. The application can also run on a mobile phone which can receive the forwarded streams of audio and video. Fig 6 shows the screenshot of the mobile version of the emergency responder application.

\section{B. Incorporating Context}

The critical issue in this system is how information is presented to different users to make timely critical decisions. M-Urgency has been developed on the Rover 2.0 platform, which maintains the context of every user, along with general context that is preserved and dynamically derived from various services described above.

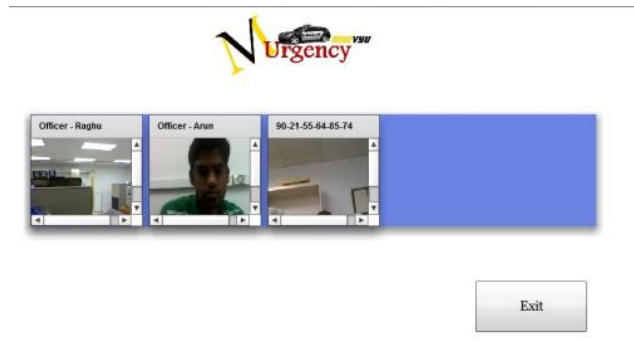

Figure 5: Screenshot of mobile version of emergency responder application.

The dispatcher and the responder are provided with contextual information, few examples of which are listed below:

- Relevant information about the user, e.g. disability or special needs, gender, age etc. so that they receive service accordingly.

- The users' real time location which is reflected on a map interface even when he/she is moving.

- As a caller is selected, the list of emergency responders is dynamically rearranged according to their distance from the caller's location or any other suitable criteria. The dispatcher can, thus, make prompt decisions in assigning the responder(s) to caller(s).

- Additional information such as weather and traffic, etc. to gather the context of the incident scene.

There have been attempts to provide location services during emergency calls [18][19]. We attempt to take it a step further by providing real-time location as the incident/user may not be static. As noted above, we consider many variables, in addition to the location, to define context pertaining to the user and the incident. The context can be better defined as more services are added to the service tier of Rover 2.0.

\section{Status and Development Details}

All the functionality of the application, the important ones being presented here, has been implemented and tested. We are in the process of deploying the pilot of the system at the University of Maryland Police Department, College Park, MD, USA. The caller applications are scheduled to be rolled out to the University community, of about 47000 people, in the beginning of Fall 2011. Additional functionalities are being regularly incorporated as the system is being used.

M-Urgency has been developed using Adobe Flex open source framework supporting desktop/laptops, android phones and tablets and blackberry tablets as of now. We are in the process of developing the versions for $\mathrm{iOS}$ devices. 
We use Adobe Flash Media Server and Adobe Flash Media Gateway for the audio and video streaming.

\section{M-Urgency and our Information Model}

The Information Model, discussed in section 3.A, can be well explained with the help of the M-Urgency system. Rover takes up the role of the "I" from Fig 1, acting as the information center for the whole system. Rover maintains static contextual information in its context storage and also gathers the dynamic context information from the clients connected to it.
- List of available responders organized such that the nearest one is pushed to the top

- The weather condition at the location. Say, if it is snowing, certain precautions need to be taken.

Based on the contextual information, the dispatcher is enabled to take very quick decisions. At the same time the dispatcher is also provided with an interface wherein he could very easily access the information and initiate the activities with mere gestures like a mouse click operation or drag-and-drop operation etc., which is important.

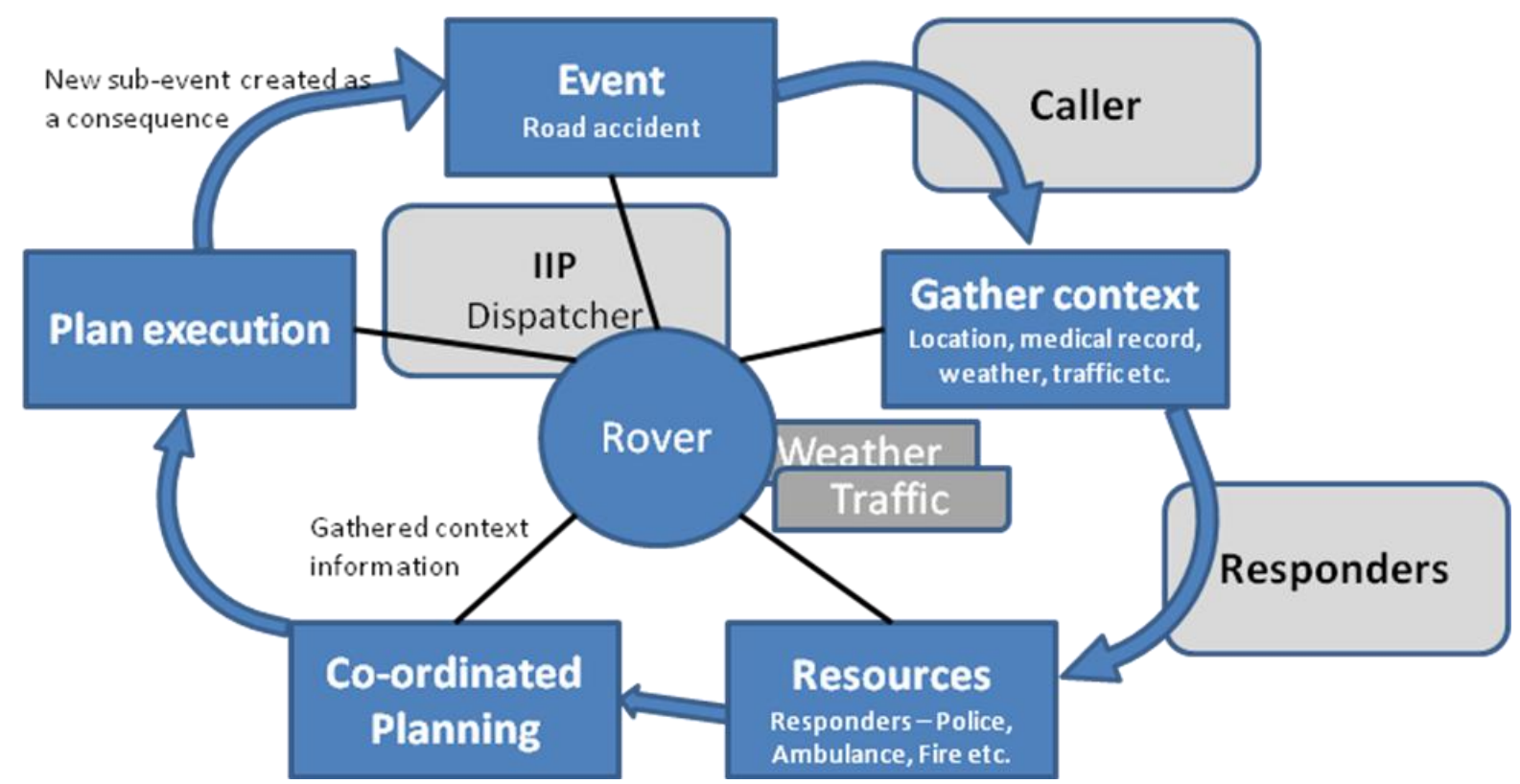

Fig 6: An instance of M-Urgency with respect to our Information Model

The role of Information Integration Point (IIP) is played by the Dispatcher Console, discussed in section 5.A. Emergency dispatchers try to make use of as much contextual information provided to them to take quick critical decisions for initiating relief activities. Say, when a call comes in from the caller application regarding a road accident, the dispatcher console is provided with contextual information, few of which are listed below:

- The caller's information like age, gender etc.

- Special needs/ disabilities that the caller may have. For example, the caller is allergic to certain medications or has poor eye sight without glasses etc.

- Callers' medical history is made available

- The video from the callers side provides a lot of visual information based on which decisions can be made

- Caller's real time location

- Traffic information around the place of accident, so that personnel dispatched to attend the accident victims can take a route accordingly
The road accident is the event in the information model that initiates the iteration. The event itself acts as a source of lot of information regarding the accident. As the event takes place, information is gathered at Rover and provided to the IIP (Dispatcher). As mentioned before, the static contextual information will be available at the Rover context storage, and the dynamic context information is gathered from various sources like the caller application itself, responder personnel applications, location server, weather server, traffic server etc.

The Dispatcher has a list of resources available, in terms of police officers, medical responders, fire responders etc. The Dispatcher initiates a coordinated planning between the police, ambulance and fire personnel. Finally the plan is executed. Each and everyone of the five nodes have to interact with Rover, either providing information or accessing information which explains why in Fig 1 we depict the inter-relationship between every node with each other and the "I" in the center. 


\section{DISCUSSION AND CONCLUSION}

We have pointed out in the paper the significance of contextual information for efficient and quick execution of relief activities in when an event, like a natural calamity or humanitarian crisis occurs. We have proposed an information model elucidating the flow, maintenance and processing of information to effectively use it in providing service.

We presented Rover 2.0, an integration platform for mobile applications that seeks to provide a mechanism to aid communication of heterogeneous information sources to all interested and authorized entities. Entities utilizing Rover add as much information as possible to aid in the fusion of information sources, including augmenting information sources with additional contextual information. Rover can provide transformations for a variety of information, from the context and content from different types of sources, into an appropriate view for first responders. It not only provides means to store and retrieve context information, but also facilitates relevant services to the applications so that the context information could be more effectively used.

We presented M-Urgency, a public safety system, developed by us to address emergency situations wherein using a smartphone an audio and video connection can immediately by established with the Police Department. Built on the Rover platform, it empowers an emergency dispatcher (Information Integration Point) with as much contextual information like the caller's details, special needs, disabilities, real-time location, audio and video feed, traffic, weather etc. that critical decisions can be taken efficiently and in a timely manner.

We believe Rover is a promising solution that would enable efficient planning and execution of relief activities as a calamity occurs. M-Urgency well supports our point in proving very effective in handling emergency situations. The initial feedback from the UM Police Department are very encouraging, though we will be performing actual empirical study on the effectiveness once the system is rolled out to the University community. We believe a variant or a progressed version of M-Urgency could well be deployed to handle emergency situations on a larger scale as calamities occur.

\section{REFERENCES}

[1] Schafer, Wendy A.; Carroll, John M.; Haynes, Steven R.; and Abrams, Stephen, "Emergency Management Planning as collaborative Community Work," Journal of Homeland Security and Emergency Management: Vol. 5: Iss. 1, Article 10.

[2] Chris P. Rainsford, Michael D. Goldsmith, and Paul Prekop. 2002. TIKI: A Trigger-Based Infrastructure for Knowledge and Information Sharing. In Proceedings of the IFIP TC5/WG5.5 Third Working Conference on Infrastructures for Virtual Enterprises: Collaborative Business Ecosystems and Virtual Enterprises (PRO-VE '02), Luis M. Camarinha-Matos
(Ed.). Kluwer, B.V., Deventer, The Netherlands, The Netherlands, 329-336.

[3] Bahrani A, Yuan, J, Emele, C.D., Masato, D, Norman, T.J, Mott D, " Collaborative and Context-Aware Planning“, IEEE Military Communications Conference, 2008. MILCOM , pp. 1-7, Nov 2008.

[4] Kikiras, Panayotis K. An Integrated Platform for Autonomic Computing for Disaster Relief Operations [online]. Journal of Battlefield Technology, Vol. 12, No. 3, Nov 2009: 29-34.

[5] Xiaodong Jiang, Nicholas Y. Chen, Jason I. Hong, Kevin Wang, Leila Takayama and James A. Landay, "Siren: Context-aware Computing for Firefighting", Pervasive Computing, pp. 87--105, 2004.

[6] Tomaszewski B, MacEachren A.M., "Geo-historical context support for information foraging and sensemaking: Conceptual model, implementation, and assessment", IEEE Symposium on Visual Analytics Science and Technology (VAST), 2010 , pp. 139-146, Oct 2010, IEEE.

[7] Alan M. MacEachren, Guoray Cai, Michael McNeese, Rajeev Sharma, and Sven Fuhrmann. 2006. GeoCollaborative crisis management: designing technologies to meet real-world needs. In Proceedings of the 2006 international conference on Digital government research (dg.o '06). ACM, New York, NY, USA, 71-72

[8] A.K. Dey and G.D. Abowd. Towards a better understanding of context and context-awareness. In CHI 2000 workshop on the what, who, where, when, and how of context-awareness, volume 4, pp. 1-6. Citeseer, 2000.

[9] C. Bolchini, C.A. Curino, E. Quintarelli, F.A. Schreiber, and L. Tanca. A data-oriented survey of context models. ACM SIGMOD Record, 36(4):19-26, 2007.

[10] M. Kaenampornpan and E. ONeill. An integrated context model: Bringing activity to context. In Proc. Workshop on Advanced Context Modelling, Reasoning and Management. Citeseer, 2004.

[11] J. Coutaz, J.L. Crowley, S. Dobson, and D. Garlan. Context is key. Communications of the ACM, 48(3):49-53, 2005.

[12] S.J.H. Yang, APM Huang, R. Chen, S.S. Tseng, and Y.S. Shen. Context model and context acquisition for ubiquitous content access in ulearning environments. In Sensor Networks, Ubiquitous, and Trustworthy Computing, 2006. IEEE International Conference on, volume 2, pages 78-83. IEEE, 2006.

[13] Roger Entner, "Smartphones to Overtake Feature Phones in U.S. by 2011 ", http://blog.nielsen.com/nielsenwire/consumer/smartphonesto-overtake-feature-phones-in-u-s-by-2011.

[14] Christian B. Almazan, Moustafa Youssef, Ashok K. Agrawala. ROVER: An Integration and Fusion Platform to Enhance Situational Awareness. In proc. IEEE International Conference on Performance, Computing and Communications 2007, IEEE (2007), 582-587.

[15] Christian B. Almazan, ROVER: Architectural Support for Exposing and Using Context. Ph.D. Thesis. University of Maryland, College Park. College Park, Maryland, United States. April 2010.

[16] Shivsubramani Krishnamoorthy and Ashok Agrawala. A context-aware framework for mobile applications. In Proceedings of the 9th international conference on Mobile systems, applications, and services, MobiSys '11, pages 403\{404, New York, NY, USA, 2011. ACM.

[17] Shivsubramani Krishnamoorthy and Ashok Agrawala. Murgency: A next generation, context-aware public Safety Application. In Proceedings of the International conference on Human Computer Interaction with Mobile Devices and Services, MobileHCI '11. ACM, 2011. 
[18] Sayed A.H, Tarighat, A.; Khajehnouri N, Network-based wireless location: Challenges Faced in Developing Techniques for Accurate Wireless Location Information, IEEE Signal Processing Magazine, Vol. 22 Issue 4, 24 40, July 2005

[19] Zagami J.M, Parl S.A, Bussgang J.J, Melillo K.D, Providing Universal Location Services Using a Wireless E911 Location Network, IEEE Communications Magazine, 66 -71, April 1998. 